\title{
Los laboratorios naturales un espacio vivo para la formación integral del estudiantado de la Universidad Comunitaria Intercultural, URACCAN
}

\author{
Oswaldo Hernández ${ }^{1}$ \\ David Díaz \\ Ariel Ruiz ${ }^{3}$ \\ Byron Ramírez ${ }^{4}$ \\ Gerardo Gutiérrez $^{5}$ \\ Néstor Morales ${ }^{6}$
}

\section{Resumen}

Tsta investigación tuvo como propósito diagnosticar el estado actual de los laboratorios naturales de EURACCAN, como espacios vivos donde se construyen y recrean conocimientos, saberes y prácticas en armonía con la madre tierra, que contribuyen a la revitalización de la identidad y cultura de los pueblos, mediante la innovación, emprendimiento, producción y comercialización, fundamentado en los lineamientos, principios y valores institucionales para el buen vivir. Los mismos se encuentran ubicados en la Costa Caribe nicaragüense, específicamente en los municipios de Waspam, Siuna, Waslala y Nueva Guinea. Se emplearon técnicas de análisis documental, observación participante, entrevistas bajo un enfoque participativo y matriz de priorización (FODA). Asimismo, se llevaron a cabo talleres participativos de discusión y análisis con las autoridades de los recintos, directores de áreas, secretarias/os académicas, coordinadores de áreas, responsables de laboratorios naturales y obreros de campo. Los resultados muestran una descripción biofísica de cada laboratorio natural, así como determinar el uso actual, las fortalezas, oportunidades, debilidades y amenazas, que permiten tomar las decisiones estratégicas oportunas $\mathrm{y}$, así mejorar en el futuro el funcionamiento donde el estudiantado, docentes, investigadores, líderes y comunitarios crean y recrean conocimientos, saberes y prácticas.

Palabras clave: laboratorios naturales; fincas experimentales; prácticas de campo; descripción biofísica; saberes y prácticas.

\footnotetext{
Médico Veterinario. Coordinador de laboratorios naturales de la Universidad de las Regiones Autónomas de la Costa Caribe Nicaraguense. Email: oswaldolabnat@uraccan.edu.ni

2 Ingeniero Agroforestal. Responsable del laboratorio natural Los Laureles de la Universidad de las Regiones Autónomas de la Costa Caribe Nicaragüense. Email: david_diazorozco@yahoo.com

3 Ingeniero Agroforestal. Responsable del laboratorio natural Awawas de la Universidad de las Regiones Autónomas de la Costa Caribe Nicaragüense. Email: ruizleivaa@yahoo.com

4 Ingeniero en Zootecnia. Responsable del laboratorio natural Bismarck Lee de la Universidad de las Regiones Autónomas de la Costa Caribe Nicaragüense. Email: byron.ramirez41@yahoo.es

5 Ingeniero Agroforestal. Responsable del laboratorio natural Snaki de la Universidad de las Regiones Autónomas de la Costa Caribe Nicaragüense. Email:. gutierrezcuthbertrene@gmail.com

6 Ingeniero en Zootecnia. Responsable del laboratorio natural Jerusalén de la Universidad de las Regiones Autónomas de la Costa Caribe Nicaragüense. Email: nestorm64@yahoo.com
} 


\section{Summary}

This research intended to diagnosticate the current state of URACCAN's natural laboratories as living spaces where knowledge and practices are built and recreated in harmony with mother earth, contributing to the revitalization of the identity and culture of the peoples, through innovation, entrepreneurship, production and marketing, based on the guidelines, principles and institutional values for the wellbeing. These labs are located on the Caribbean Coast of Nicaragua, specifically in the municipalities of Waspam, Siuna, Waslala and Nueva Guinea. The methodology is based on documentary analysis techniques, participant observation, interviews with a participatory approach and prioritization matrix (SWOT). Likewise, we carried out participative workshops for discussion and analysis with the authorities of the campus, directors of areas, academic secretaries, coordinators of areas, responsible of natural laboratories and field workers. The results show a biophysics description of each natural lab, and it also determine the current use, strengths, opportunities, weaknesses and threats, that allows to make the right strategic decisions and thus, improve in the future the performance in which the students, teachers, researchers, leaders and community create and recreate knowledge and practices.

Keywords: Natural laboratories; experimental farms; field practices; biophysics description; knowledge and practices.

\section{Introducción}

T a Universidad de las Regiones Autónomas de Lla Costa Caribe Nicaragüense como institución académica de Educación Superior, comunitaria intercultural y comprometida con la calidad en todos los ámbitos de su quehacer, ve la necesidad de trabajar en la articulación de todas las áreas como estrategia importante en el desarrollo institucional. Tal propósito, sólo se verá alcanzado, si se aplican las estrategias adecuadas y pertinentes para fortalecer las relaciones entre la teoría, práctica e investigación, para que retroalimenten e interactúen en los procesos de formación, investigación y sostenibilidad.

En este marco, los cinco laboratorios naturales, ubicados en los recintos universitarios de Bilwi, Nueva Guinea y Las Minas, cobran una gran signifi- cancia como asignatura pendiente de articulación a trabajar en la URACCAN. Puesto, que son considerados espacios vivos donde se construyen y recrean conocimientos, saberes y prácticas en armonía con la Madre Tierra; que contribuyen a la revitalización de la identidad y cultura de los pueblos de la Costa Caribe mediante la innovación, emprendimiento, producción y comercialización fundamentado en los lineamientos, principios y valores institucionales para el Buen Vivir.

Por ello, como ejercicio efectivo de la voluntad política de Rectoría en el mejoramiento de estos espacios, desde el año 2014 se da inicio a visitas in situs para determinar el estado actual y la articulación con la docencia, producción sostenible y comercialización de los productos. Posteriormente se llevó a cabo la presente investigación con el fin de diagnosticar la situación actual de los laboratorios naturales. En el cual se pudo describir cada laboratorio, determinar el uso actual y analizar las fortalezas, oportunidades, debilidades y amenazas para poder tomar las decisiones estratégicas oportunas y mejorar en el futuro.

\section{Revisión de literatura}

Etimológicamente el concepto diagnóstico proviene del griego, tiene dos raíces, día- que es a través de, y gignoskein- que es conocer. Así etimológicamente, significa "conocer a través de". El concepto de este significado es la identificación de la naturaleza o esencia de una situación o problema y de la causa posible o probable del mismo, es el análisis de la naturaleza de algo (Vera, 2006). También, se define diagnóstico como "el proceso que se realiza en un objeto determinado, generalmente para solucionar un problema". El diagnóstico, consta de varias etapas, dialécticamente relacionadas, que son: evaluación, procesamiento mental de la información, intervención y seguimiento.

Según Gutiérrez (2006), el diagnóstico se fundamenta en observaciones y registro de información de campo a través de un equipo técnico interdisciplinario y la interacción participativa con el productor y su familia. La información para el diagnóstico responde a las siguientes preguntas: ¿Qué existe en los laboratorios naturales (Finca)?, referido a: usos de la tierra, factores determinantes de productividad como fertilidad de suelos, pendientes del terreno; sistemas agroforestales actuales y sus componentes. ¿Cuál es el conocimiento acerca del establecimiento, productividad, manejo, 
usos, aprovechamiento del Sistemas Agroforestales (SAF) y de sus componentes? ¿Cuáles son las limitantes, potencialidades y proyecciones relacionadas con el sistema agroforestal?

\section{Descripción biofísica del área}

El Instituto Interamericano de Cooperación para la Agricultura (IICA, 1992) plantea que la descripción biofísica, es la descripción de las características biológicas y físicas de los suelos en cada área e incluye la:

- Localización y extensión: Ubica la superficie del suelo geográficamente, con las coordenadas y define la extensión del terreno en unidades de superficie.

- Geología: estudia el origen del suelo, formación y evolución de la tierra, estructura y materiales que lo componen.

- Relieve y topografía: le da connotación al suelo de acuerdo a las características fisiográficas y formaciones geológicas.

- Hidrografía: Estudia las cuencas, tributarios y ríos existentes en la zona de estudio.

- Características bioclimáticas: Describe el comportamiento meteorológico de la zona, incluye régimen de precipitaciones y de no precipitaciones, régimen térmico, humedad relativa, brillo solar, velocidad y dirección del viento.

- Suelos: caracteriza el suelo de la zona según el origen y propiedades del mismo.

Por tanto, la caracterización biofísica de una finca implica ubicar, delimitar, identificar, describir todos los componentes que integran la finca con la finalidad de realizar un ordenamiento de la misma bajo criterios ecológicos, productivos y didácticos.

\section{Uso del suelo}

Uno de los recursos naturales más importante es el suelo. El uso del mismo, se refiere a los diferentes modos de utilización que se hacen o deben hacerse del terreno para los servicios o funciones urbanas o regionales (Ministerio de Vivienda y Asentamientos Humanos, 1982); mientras que la FAO (1999) plantea que es el total de los arreglos, las actividades y los insumos que las personas llevan a cabo en un determinado tipo de cobertura terrestre". En este sentido Castro y Requene (2015) consideran, que el tema de uso y explotación de tierras en Nicaragua juega un papel sumamente importante dentro de la economía. Por lo tanto, es necesario hacer políticas y normas que apunten al desarrollo integral, económico y agrícola de la sociedad. Esto implica, la necesidad de un marco de posibilidades para mejorar la calidad de vida de hombres y mujeres en igualdad de condiciones.

\section{Análisis FODA o Matriz DAFO}

Espinosa (2013) considera que la matriz de análisis DAFO o FODA, es una conocida herramienta estratégica de análisis de la situación de la empresa u organización. El principal objetivo de aplicar la matriz DAFO en una organización, es ofrecer un claro diagnóstico para poder tomar las decisiones estratégicas oportunas y mejorar en el futuro, su nombre deriva del acrónimo formado por las iniciales de los términos: debilidades, amenazas, fortalezas y oportunidades. Esta matriz permite, identificar tanto las oportunidades como las amenazas que presentan el mercado, y las fortalezas y debilidades que muestra la empresa u organización.

\section{Materiales y método}

El diagnóstico de los laboratorios naturales de la URACCAN se realizó en las Regiones Autónomas de la Costa Caribe, en los municipios de Waspam, Siuna, Waslala y Nueva Guinea. El territorio cuenta con una gran diversidad étnica, cultural, lingüística, geográfica y de biodiversidad. Donde conviven diferentes pueblos: miskitos, sumo-mayangnas, ulwas, rama, creol, garífuna, y mestizos, con memorias colectivas, historias, cultura e idiomas, que los diferencian entre sí del resto de la población. Uno de los ejes del capital social que se promueve es el de juntos comparten el cual está vinculado con la importancia de las historias y vivencias comunes en la construcción del tejido social comunitario y con la cosmovisión que se tiene como pueblo.

La mayor parte de la población costeña depende económicamente de las actividades en los siguientes sectores: comercio y servicios, agrícola, pesquero, y silvopastoril. Goza de un régimen de Autonomía, el cual fue creado mediante la Ley 28: Estatuto de la Autonomía de las Regiones de la Costa Atlántica de Nicaragua, la que surge como el modelo de organización político territorial para reconocer los derechos históricos de los pueblos.

El paradigma en que se basa esta investigación es cualitativo y cuantitativo sustentado en un diseño descriptivo. Se emplearon técnicas como: análisis documental, observación participante, entrevistas bajo un enfoque participativo y matriz 
de priorización (FODA). Asimismo, se llevaron a cabo talleres participativos de discusión y análisis con las autoridades de los recintos, directores de áreas, secretarias/os académicas, coordinadores de áreas, responsables de laboratorios naturales y obreros de campo.

Para recopilar la información del diagnóstico de los laboratorios naturales se elaboraron y aplicaron los instrumentos respectivos: guía de preguntas, lista de cotejo y guía metodológica de visitas de acompañamiento y guía de diagnóstico. Los que permitieron recopilar los datos necesarios para cumplir con los objetivos propuestos.

\section{Resultados y discusión}

\section{Descripción biofísica de los laboratorios naturales de URACCAN}

La URACCAN, en su desarrollo y fortalecimiento institucional, adquirió como parte de su pensamiento, planificación y gestión de cinco laboratorios naturales. Estos fueron concebidos como espacios vivos donde se construyen y recrean conocimientos saberes y prácticas, en armonía con la madre tierra, que contribuyen a la revitalización de la identidad y cultura de los pueblos mediante la innovación, emprendimiento, producción y comercialización, fundamentado en los lineamientos, principios y valores institucionales para el buen vivir.

Se ubican en la Costa Caribe nicaragüense, específicamente en los municipios de Waspam, Siuna, Waslala y Nueva Guinea. La adquisición de los mismos comienza en el 2000 con el Laboratorio Los Laureles (Siuna), seguidamente Snaki (Waspam) en el 2003, posteriormente Jerusalén (Nueva Guinea) en el 2007 y en el 2008 Awawas (Siuna). Concluyendo, en el 2013 con la compra del laboratorio natural Bismarck Lee en Waslala. Véase figura 1.

En su conjunto forman parte del patrimonio de la Universidad y de la Costa Caribe nicaragüense como espacios vivos de trabajo donde el estudiantado, docentes, investigadores, líderes y comunitarios crean y recrean conocimientos, saberes y prácticas.

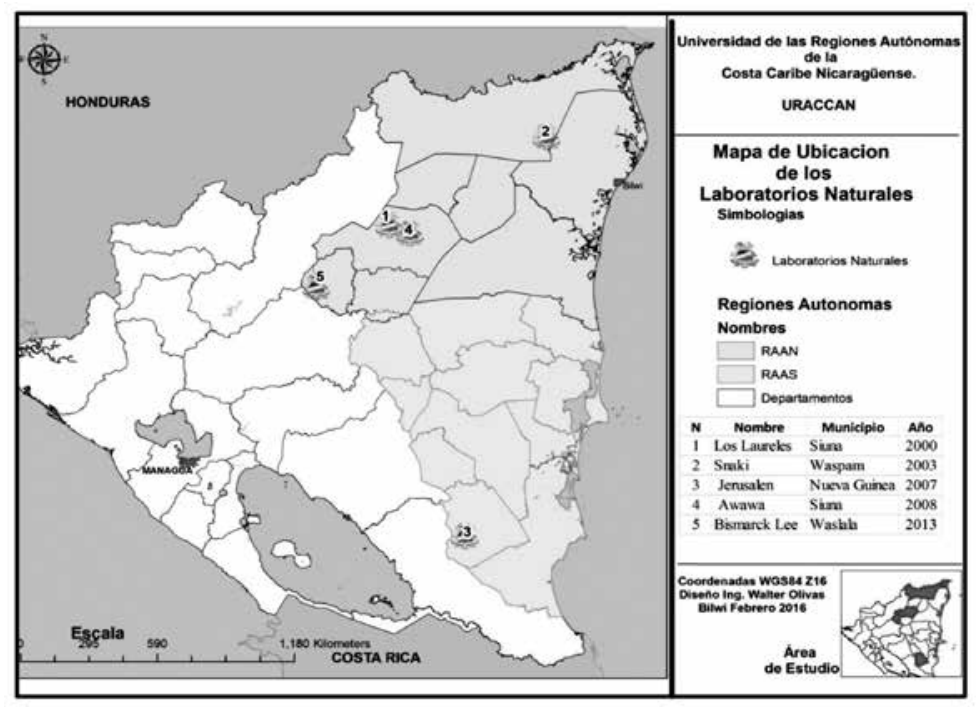

Figura no. 1. Ubicación de los Laboratorios Naturales de URACCAN. CISA-IREMADES (2015).

La URACCAN dispone en su totalidad 251 ha en la Costa Caribe nicaragüense para la formación integral del estudiantado, generación de conocimientos, establecimiento de sistemas productivos agroforestales, agrícolas, pecuarios e industriales, así como la comercialización de productos y la protección de los recursos naturales. 
Tabla no. 1. Extensión territorial, pluviosidad y topografía de los laboratorios naturales de URACCAN

\begin{tabular}{|c|l|c|c|}
\hline Nombre del Laboratorio Natural & \multicolumn{1}{|c|}{ Extensión Territorial $(\mathrm{Ha})(\mathrm{Mz})$} & Pluviosidad mm de agua anual & Altura promedio (msnm) \\
\hline Los Laureles & $48 \mathrm{Ha} ; 67.8 \mathrm{Mz}$ & 2800 & 114 \\
\hline Awawas & $\begin{array}{l}17,91 \mathrm{Ha} 102,09 \\
25.1 / 4 \mathrm{Mz} 143,9\end{array}$ & 2800 & 157 \\
\hline Bismarck Lee & $\begin{array}{l}36,18 \mathrm{Ha} \\
51,01 \mathrm{Mz}\end{array}$ & 3270 & 680 \\
\hline Snaki & $\begin{array}{l}100,70 \mathrm{Ha} \\
142 \mathrm{Mz}\end{array}$ & 2970 & 25,5 \\
\hline Jerusalén & $\begin{array}{l}48,2 \mathrm{Ha} \\
67,95 \mathrm{Mz}\end{array}$ & 3004 & 188 \\
\hline Total & $\begin{array}{l}250,99 \mathrm{Ha} \\
353,9 \mathrm{Mz}\end{array}$ & $2800-3270$ & $25-680$ \\
\hline
\end{tabular}

La precipitación anual promedio en Siuna es de 1,800 $\mathrm{mm}$ a 2,800 $\mathrm{mm}$ anuales. En algunos sitios se estiman precipitaciones de 3,200 $\mathrm{mm}$, la estación seca corresponde a los meses de febrero, marzo y abril. obtiene su máximo pico en el mes de junio, siendo la época de lluvia de mayo a noviembre. El clima es de tipo húmedo tropical monzónico (Alcaldía de Siuna, 2015). Los datos que se reportan son similares a los registrados por (Díaz, 2014) desde la estación meteorológica del Centro Agronómico Tropical de Investigación y Enseñanza (CATIE) con promedio mensual de precipitaciones de $202.02 \mathrm{~mm}$, y que las máximas precipitaciones se registran en los meses de junio a octubre y las mínimas en los meses de marzo a abril.

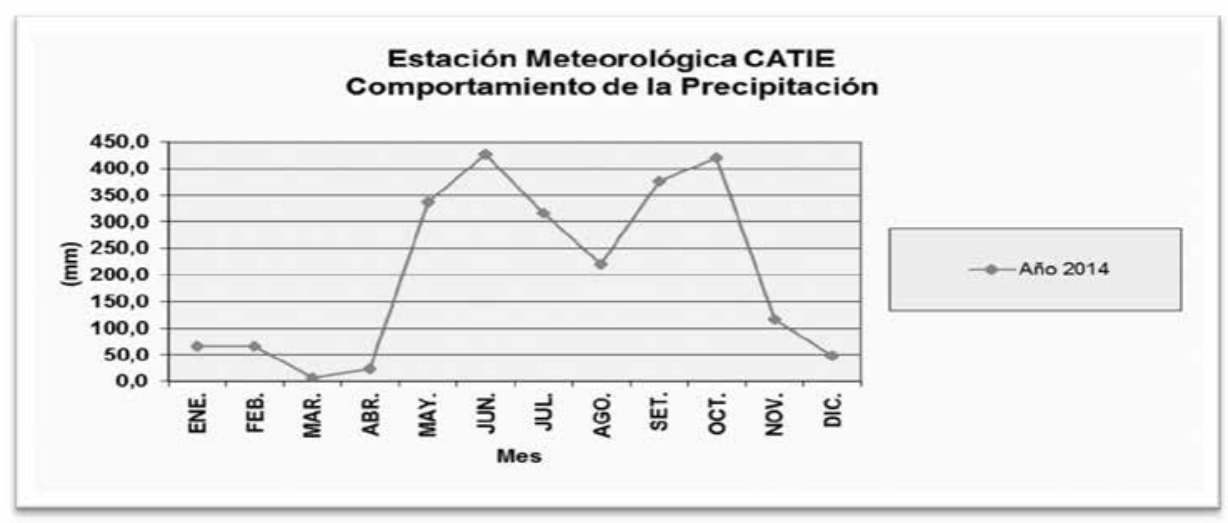

Figura no. 2. Comportamiento de las precipitaciones en los laboratorios naturales Los Laureles y Awawas, según CATIE (2014). Siuna-Campbell Scientific CR800.

Gutiérrez (2014) reporta que en Waspam se registran precipitaciones de 2,970 $\mathrm{mm}$, siendo los meses de menor precipitación marzo y abril con $180 \mathrm{~mm}$ y el período de mayor pluviosidad de junio a octubre con más de $300 \mathrm{~mm}$. En este período se registra el 80\% del total de lluvia anual.

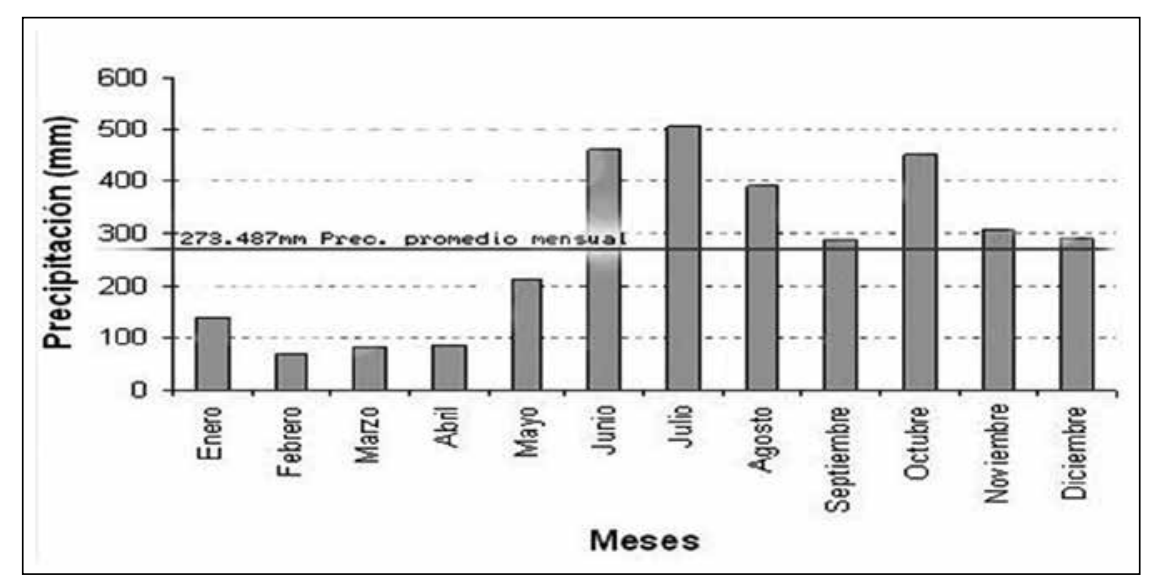

Figura no. 3. Comportamiento de las precipitaciones en el laboratorio natural Snaki, según INETER (2000- 2007) 
Nueva Guinea, se ubica entre las regiones del trópico húmedo, por lo que el clima es de selva tropical con precipitación promedio anual de $3.004 \mathrm{~mm}$, que pueden variar entre los 3,000 $\mathrm{mm}$ a 3,008 $\mathrm{mm}$ de lluvia por año y $500 \mathrm{~mm}$ mensual. La estación lluviosa se presenta desde mayo hasta enero y la seca de febrero a abril. En la figura 4 se muestra que el período más lluvioso es de junio hasta agosto y los menos lluviosos de febrero a abril (INETER, 2009).

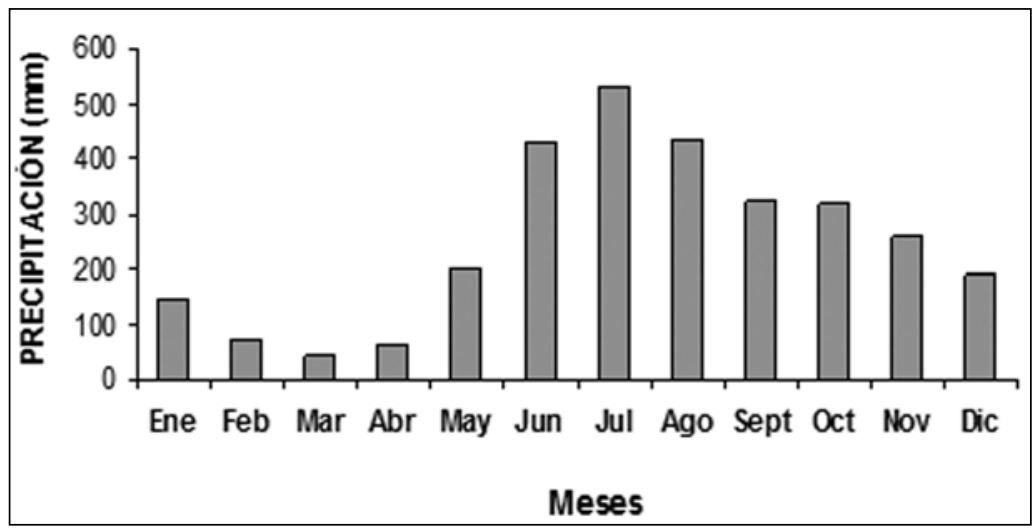

Figura no. 4. Comportamiento de las precipitaciones en el laboratorio natural Jerusalén (INETER, 2009)

El clima de Waslala, está en la categoría de trópico húmedo, con precipitación promedio anual de 3,270 $\mathrm{mm}$ de lluvia, con mínimas de 3,000 $\mathrm{mm}$ y máximas de 3,540 $\mathrm{mm}$ de lluvia, el período lluvioso en el territorio de Waslala, inicia desde mayo hasta noviembre y el período menos lluvioso, es desde febrero a abril, siendo los meses más lluviosos junio, julio y agosto y los menos lluviosos marzo y abril.

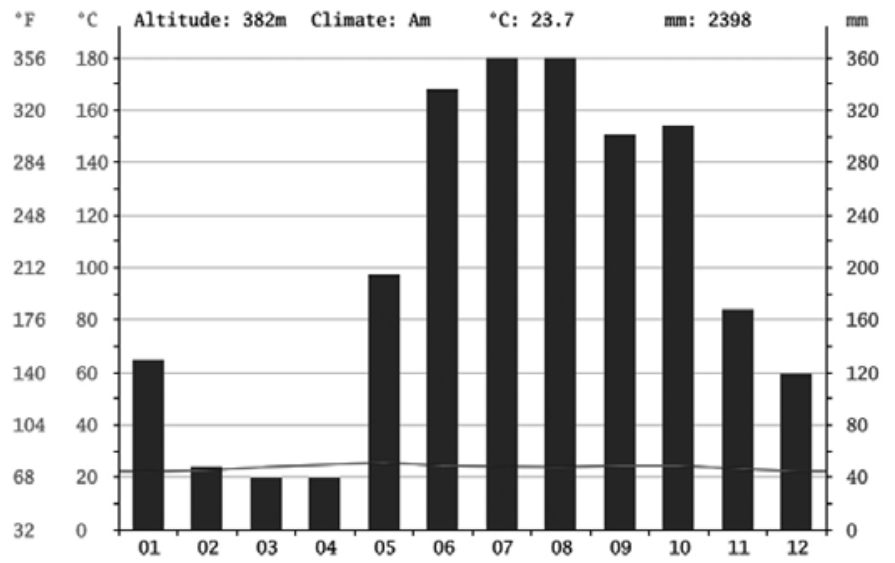

Figura no. 5. Comportamiento de las precipitaciones en el laboratorio natural Bismarck Lee. Según CLIMATE- DATA-ORG (2015).

De acuerdo al régimen pluvial registrado en diferentes zonas de la Costa Caribe, se registra clima tropical húmedo monzónico. Los suelos en la Costa Caribe son variados como se puede observar en la tabla 2, encontrándose en el laboratorio natural Jerusalén suelos arcillosos y francos arcillosos, esto indica que son suelos que retienen alto grado de humedad y que son muy susceptibles a la erosión (Calero, 2010). En Snaki el suelo es franco y franco arenosos óptimos para la agricultura, también es uno de los indicadores de suelo con elevada productividad (Wilson y Sujo, 2013). En Siuna los suelos son arcillosos, limosos y franco arcilloso, con drenaje bueno y moderado, con buena cantidad de materia orgánica y PH ligeramente ácido a muy ácido (Alcaldía de Siuna, 2015), y en Waslala se encuentran suelos franco arcilloso y arcilloso, textura del subsuelo de arcilloso a muy arcilloso (Alcaldía de Waslala, 2012). 
Tabla no. 2. Caracterización de los suelos y el uso actual de los laboratorios naturales de URACCAN.

\begin{tabular}{|c|c|c|c|}
\hline $\begin{array}{c}\text { Nombre del } \\
\text { LN }\end{array}$ & Tipo de suelo & Uso actual & Rubros productivos \\
\hline Los Laureles & $\begin{array}{l}\text { arcilloso, limosos y } \\
\text { franco arcilloso } \\
\text { (Bueno para cultivos) }\end{array}$ & $\begin{array}{|ll|}\text { Sistema agroforestal. } & \\
\text { Silvopastoril, cultivos } & \text { varios, } \\
\text { conservación de } & \text { recursos } \\
\text { naturales y pecuario. } & \\
\end{array}$ & \begin{tabular}{|lr} 
Café, & Cacao, \\
Musáceas, & Granos \\
básicos, & ganado \\
bovinoy porcino.
\end{tabular} \\
\hline Awawas & $\begin{array}{l}\text { Arcilloso, limoso y franco } \\
\text { arcillosos } \\
\text { Muy bueno para cultivos) }\end{array}$ & $\begin{array}{l}\text { Sistema } \\
\text { conservación y pecuario }\end{array}$ & $\begin{array}{l}\text { Granos básicos, } \\
\text { crianza de cerdos, } \\
\text { Zoocriaderos y } \\
\text { forestal. }\end{array}$ \\
\hline $\begin{array}{l}\text { Bismarck } \\
\text { Lee }\end{array}$ & $\begin{array}{l}\text { Franco arcilloso y arcilloso } \\
\text { (Regular para cultivos y } \\
\text { muy bueno para lo } \\
\text { forestal) }\end{array}$ & $\begin{array}{l}\text { Sistema } \quad \text { Agroforestal } \\
\text { conservación, cultivos varios }\end{array}$ & $\begin{array}{ll}\text { Café, } & \text { Cacao, } \\
\text { Granos } & \text { básicos, } \\
\text { musáceas y } & \text { Forestal }\end{array}$ \\
\hline Snaki & $\begin{array}{l}\text { Franco arenoso } \\
\text { (Muy bueno para el } \\
\text { cultivoy otros usos) }\end{array}$ & $\begin{array}{l}\text { Sistema } \quad \text { agroforestal, } \\
\text { silvopastoril, cultivos varios y } \\
\text { pecuario. }\end{array}$ & $\begin{array}{l}\text { Café, Cacao, } \\
\text { Musáceas, Granos } \\
\text { básicos, ganado } \\
\text { bovinoy porcino. }\end{array}$ \\
\hline Jerusalén & $\begin{array}{l}\text { Franco arcilloso y arcilloso } \\
\text { (Regular para cultivos y } \\
\text { muy bueno para forestal, } \\
\text { pecuario) }\end{array}$ & $\begin{array}{l}\text { Sistema agroforestal. } \\
\text { Silvopastoril, cultivos varios, } \\
\text { conservación y pecuario. }\end{array}$ & $\begin{array}{l}\text { Cacao, } \\
\text { bovino, } \\
\text { conejo, } \\
\text { granos } \\
\text { forestal }\end{array}$ \\
\hline
\end{tabular}

Los sistemas productivos establecidos para la producción de alimentos en los diferentes laboratorios naturales son agroforestales, forestales y agropecuarios, siendo los rubros de mayor importancia el café, cacao, musáceas, granos básicos, fruticultura y la ganadería de tipo extensiva. La comunidad estudiantil es la que más utilidad les da a los laboratorios naturales para la generación de conocimientos, saberes y prácticas, así como la producción de alimentos de origen vegetal y animal.

La Tabla 3 muestra los resultados del análisis de la Matriz FODA aplicada para la evaluación de las principales fortalezas, oportunidades, debilidades y amenazas de los laboratorios naturales de URACCAN, dejando visible el camino a seguir para emprender el programa estratégico aprovechando las oportunidades existentes enfocadas en disminuir al mínimo el efecto negativo de las amenazas. 
Tabla no. 3. Matriz FODA de los Laboratorios Naturales de URACCAN, 2015.

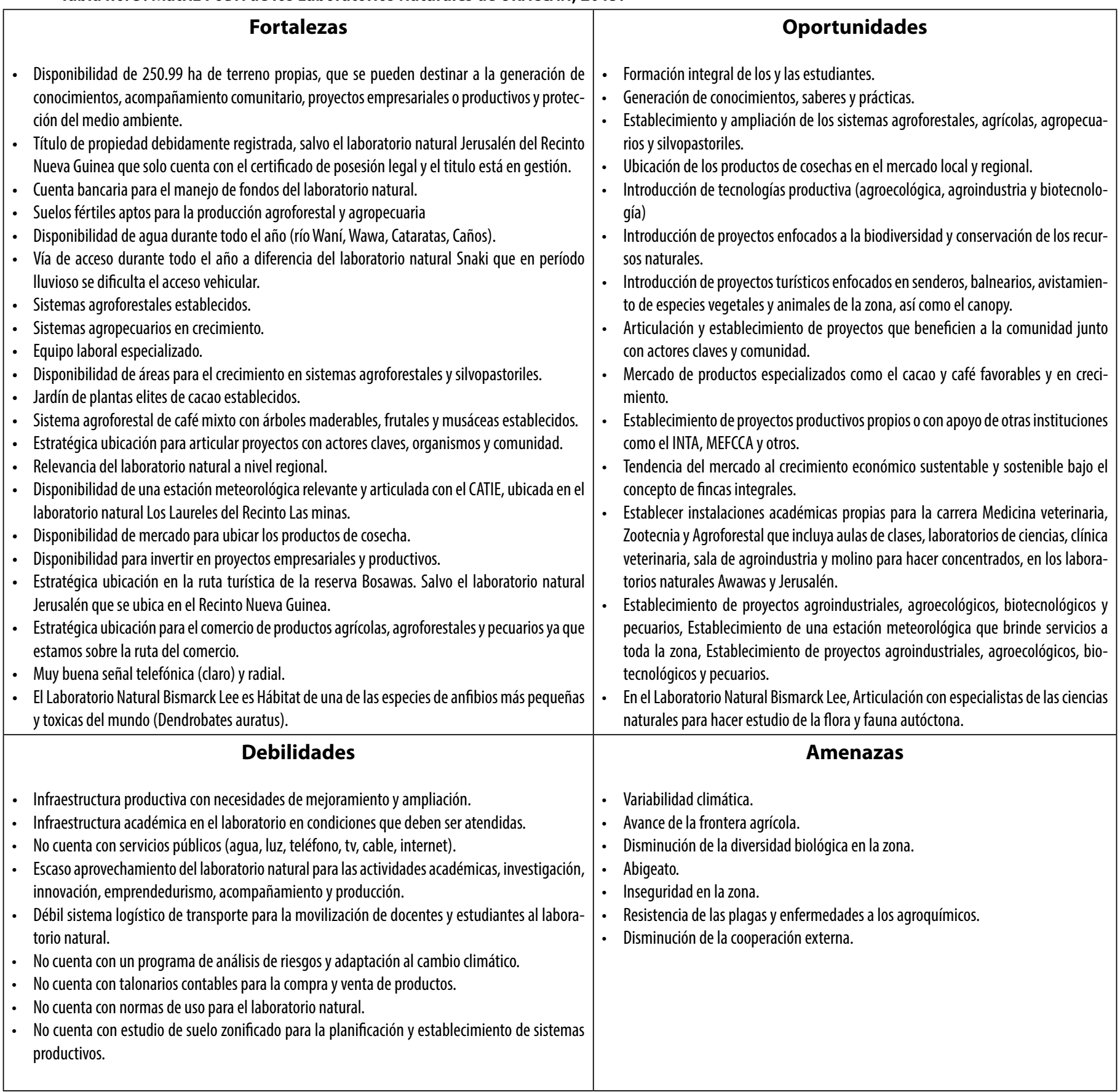

\section{Conclusiones}

La URACCAN cuenta con 250.99 Ha de terreno, ubicadas estratégicamente desde la Costa Caribe Norte hasta la Costa Caribe Sur, las cuales son destinadas para la enseñanza y aprendizaje, generación de conocimientos, saberes y prácticas, acompañamiento comunitario, aprovechamiento productivo con sistemas agroforestales, silvopastoriles y agroecológicos, comercialización de productos y protección de la madre tierra. 
Los laboratorios naturales cuentan con topografía ondulada y suelos diversos que van desde franco a franco arenosos, arcillosos, franco arcillosos y limosos donde el nivel de fertilidad es variable y por sus características los suelos del laboratorio natural Snaki en Waspam, Los Laureles en el Siuna y Jerusalén en Nueva Guinea, tienen bondades para establecer sistemas agroforestales, silvopastoriles, agroecológicos y pecuarios. Las bondades del suelo en el laboratorio natural Bismarck Lee de la extensión Waslala, son para los sistemas productivos agroforestales, agroecológicos y floricultura dado a la topografía inclinada del terreno que no da lugar al establecimiento del sistema productivo pecuario.

El laboratorio natural Awawas del Recinto Las Minas, tiene suelos de tendencia limosos caracterizados por drenaje regular y formación de terrones fáciles de trabajar lo que permite establecer sistemas productivos agroforestales y agroecológicos permitiendo el establecimiento de otros sistemas productivos, pero por razones de ubicación no se pueden implementar. Cuentan con grandes oportunidades de crecimiento respaldados por la tenencia de títulos de propiedad registrados, salvo el de Jerusalén en Nueva Guinea que solo cuenta con un certificado de posesión legal (Titulo en gestión); Además tienen una cuenta bancaria que sirve para los movimientos monetarios propios del laboratorio natural, salvo Jerusalén en Nueva Guinea y Bismarck Lee en la Extensión Waslala que no lo han hecho, pero está en gestión.

La ubicación es estratégica ya que se encuentran en la ruta del comercio de productos agrícolas y pecuarios, salvo Snaki que presenta dificultades en período lluvioso porque son caminos sin mantenimiento, pero aún así entran los comerciantes de granos básicos hasta la Comunidad de Mos; además Los Laureles, Awawas y Bismarck Lee se encuentran en el área de amortiguamiento de la reserva Bosawas en la ruta perfecta para incursionar en el mercado del turismo ecológico. Cuenta con gran fortaleza representada por los recursos naturales existentes como la disponibilidad de agua (Rio Wawa, Wani, Cascadas en "Bismarck Lee" y el Caño Siuna), bosques establecidos y naturales, diversidad de fauna silvestre y hábitat de una de las especies de anfibios más pequeños y venenosos como la rana dardo venenoso (Dendrobates Auratus).

Los laboratorios naturales cuentan con sistemas agroforestales establecidos (cultivos de café marsellesa o robusta mixto, con otras especies), sistemas silvopastoriles establecidos (pastos naturales o mejorados mixtos con otras especies), sistemas agroecológicos (cultivos mixtos de maíz, frijoles yuca, gandul y otros abonos verdes). Los laboratorios naturales y las políticas de articulación de todas las áreas orientadas desde la rectoría favorecen la formación académica de los y las estudiantes por ende el mejoramiento de la calidad de vida de los pueblos de la Costa Caribe Nicaragüense para el buen vivir.

El laboratorio natural Los Laureles, Snaki y "Bismarck Lee" tiene innumerables recursos que se pudieran aprovechar para el desarrollo de programas turísticos y aprovechar la ubicación en la zona de amortiguamiento de la reserva biosfera de Bosawas. Los laboratorios naturales cuentan con el talento humano permanente y especializado para el buen desarrollo y desempeño de los proyectos y programas planificados.

Por lo tanto, se recomienda: crear la cuenta bancaria para el laboratorio natural "Bismarck Lee" de la Extensión Waslala y el laboratorio natural Jerusalén del Recinto Nueva Guinea; realizar estudio de suelos en todos los laboratorios naturales, análisis detallado por unidades de terreno homogéneas ya que servirá para la elaboración de la propuesta para el plan de desarrollo; dar seguimiento a la gestión de solicitud de título de propiedad para el laboratorio natural Jerusalén del Recinto Nueva Guinea; aplicar metodologías enfocadas al incremento de la utilización de los laboratorios naturales como ambientes para la enseñanza y aprendizaje, generación de conocimientos, creación y recreación de conocimientos, innovación, emprendedurismo, aplicación de nuevas tecnologías productivas, producción sostenible con enfoque a la protección del medio ambiente y los recursos naturales.

Diseñar un programa que garantice la movilización de docentes y estudiantes hasta los laboratorios naturales para el desarrollo de actividades prácticas, establecimiento de ensayos, proyectos productivos y elaboración de estudios científicos; elaborar un programa detallado para el crecimiento o remodelación de infraestructuras académicas, productivas, deportivas, turísticas, alojamiento estudiantil y cercas perimetrales; elaborar y gestionar proyectos de inversión con fondos de cooperación; y construir el Plan de Desarrollo Quinquenal para los laboratorios naturales. 


\section{Bibliografía}

Alcaldía de Siuna (2015). Ficha técnica del Municipio. RACCN, Nicaragua.

Alcaldía de Waslala (2012). Ficha técnica de Waslala. RACCN. Waslala, Nicaragua.

Calero (2010). Diagnóstico de la finca experimental URACCAN referente a la caracterización de los suelos. RACCS, Nicaragua.

Castro, A. y Requene, G. (2015). Uso y explotación de las tierras en Nicaragua. Revista electrónica de investigación en ciencias económicas REICE. Vol.3 (5). 191:204

CATIE (2014). Estaciones meteorológicas de Nicaragua.

CISA_IREMADES (2015). Mapa de ubicación de los laboratorios naturales de URACCAN. Bilwi, Nicaragua.

CLIMATE-DATA- ORG (s.f.). Clima: Nueva Guinea. Consultado el martes 11 de octubre del 2016. Recuperado de: http://es.climate-data.org/ location/3088o/CLIMATE-DATA- ORG

Díaz, M. (2014). Informe técnico del laboratorio natural Los Laureles. Siuna, RACCN, Nicaragua.

Espinosa, R. (2013). La matriz DAFO-FODA. España. Recuperado de robertoespinosa. es/2013/o7/29/la-matriz-dafo-foda.
FAO (1999). Categorías de uso de los terrenos reconocidos en el censo mundial de la agricultura. Recuperado de: https://es.wikipedia.org/wiki/ Usos_del_suelo.

Gutiérrez, B. (2006). Diagnóstico y diseño participativo en sistemas agroforestales. Manual y guías de campo. Primera edición. Santa fé de Bogotá, Colombia.

Gutiérrez, G. (2014). Informe técnico del laboratorio natural Snaki. Waspam, RACCN, Nicaragua.

IICA (1992). Estudio de suelos áreas semiáridas de la región del trifinio. Proyecto de desarrollo e integración regional. Chiquimula, Guatemala.

INETER (2009). Caracterización del clima en la RACCS. Municipio de Nueva Guinea, Nicaragua.

Ministerio de Vivienda y Asentamientos $\mathrm{Hu}-$ manos (1982). Decreto No. 504. Reglamento de zonificación y uso del suelo para el área del municipio de Managua. Publicado en La Gaceta No. 110, del 12 de mayo de 1982. Nicaragua. Recuperado de: http://legislacion.asamblea. gob.ni/normaweb.nsf/(\$All)/8379oEB4512BE825062570A100579127?OpenDocument

Vera, M. (2006). Conceptos de diagnóstico. San Carlos, MX.

Wilson, F. y Sujo, J. (2013). Suelos de la finca académica Snaki. RACCN. Bilwi, Nicaragua. 